\title{
Detection of the chaotic flow instability in a natural convection loop using the recurrence plot analysis and the nonlinear prediction
}

\author{
Hiroyuki NISHIKAWA*, Kazuyuki MATSUMURA**, Shigeki OKINO**, \\ Takashi WATANABE* and Fujio SUDA** \\ *Graduate School of Information Science, Nagoya University \\ Furo-cho, Chikusa-ku, Nagoya-shi, 464-8061, Japan \\ E-mail: nishikawa@vi.cs.is.nagoya-u.ac.jp \\ **Department of Resources and Environment Science, Tokai University \\ 4-1-1 Kitakaname, Hiratsuka-shi, Kanagawa 259-1292, Japan
}

Received 8 November 2015

\begin{abstract}
An experiment and a time series analysis have been made on the flow in a rectangular natural convection loop. It was found that the flow reversal occurs in a certain range of the heat flux and Reynolds number, which depends on the cooling water temperature. Based on time series temperature data of the system, an attractor in a state space has been reconstructed. Geometrical structures and orbital instability for this attractor were evaluated by the recurrence plot analysis and a nonlinear prediction by the method of local linear approximation. A nonlinear prediction was applied to the turbulence time series. As a result, a recurrence plot of the predicted time series obtained from the turbulence data has characteristics similar to those of the measured of the unstable flow. This fact suggests that, even when an apparent turbulent flow is observed, a transition to the unstable flow may occur. In order to confirm this conjecture, we have performed another experiment with the addition of a disturbance to the loop. The experimental results are consistent with the chaos analysis. We have successfully shown the validity of the nonlinear prediction and its recurrence plot as a means to evaluate dynamical flow instability.
\end{abstract}

Key words: Natural convection, Flow instability, Chaos, Turbulence, Recurrence plot, Nonlinear prediction

\section{Introduction}

Natural convection loops are an efficient heat transfer equipment. They have many applications for the purpose of cooling in industrial processes because of their efficient heat removal without a pump. There is, for example, permafrost protection, the production of geothermal energy, solar heating, cooling of the CPU, the emergency cooling system of nuclear reactors and so on. There have been reported many studies on natural convection loops since two early analyses by Keller (1966) and Welander (1967) were performed. As an early experiment, Creveling, et al. (1975) clearly observed the flow instabilities of water in a toroidal natural convection loop. Misale et al. (2007, 2012) reported experimental studies in a rectangular loop. The circulation flow in a natural convection loop is driven by buoyancy forces which have resulted from density differences created by heating and cooling of the working fluid. In spite of the simplicity of its operating principle, the flow shows complex behavior. In particular, an irregular reversal flow has been also found the early studies. Its reversal flow is an unstable chaotic phenomenon (Nishikawa, et al., 2009; Nishikawa et al., 2012). Flow in a loop for industrial device is turbulent one in general. In this case, a cavitation flow, causing damage to the tube wall is a problem to be addressed. Another possible problem is that the chaotic flow due to an external disturbance induces an accident. Experimentally, the reversal of the flow is likely to occur in a rectangular loop than a toroidal loop (Suda, 1997). Moreover, in the case that the kinematic viscosity of the working fluid is large, and heat transfer coefficient of the tube wall is high, the occurrence of the unstable flow is suppressed (Jiang and Shoji, 
2003; Suda et al., 2005).

The unstable behavior of the flow has been much investigated theoretically or experimentally, but there have been only a few studies on the behavior of the unstable flow from the standpoint of chaos analysis. In particular, the process of transition from the unstable flow to turbulence is not clearly understood yet. In order to predict the flow instability due to disturbances of accidents such as earthquake, it is necessary the development of methods, which can be evaluated the stability of the observing data.

In the present study, an experiment and a time series analysis have been made on the flow in a rectangular natural convection loop. Especially, we paid much attention to the flow instability extracted from reconstructed attractors in state spaces. Time series data of temperature oscillation were measured for under various heat fluxes and cooling water temperature conditions. Based on these data, an attractor in high-dimensional space has been reconstructed by obtaining optimum delay time (Takens, 1981). Then, by using the recurrence plot analysis, chaotic instability was identified from geometrical structure of the attractor (Casdagli, 1997; Marwan, et al., 2002, 2007). Furthermore, measured time series were examined whether or not to destabilize using a nonlinear prediction by the method of local linear approximation (Sano and Sawada, 1985; Ikeguchi and Aihara, 1995). In addition, we have performed another experiment with an addition of disturbance to the loop.

\section{Nomenclature}

$c$ : Specific heat capacity, $\mathrm{J} /(\mathrm{kg} \cdot \mathrm{K})$

$g$ : Gravitational acceleration, $9.80 \mathrm{~m} / \mathrm{s}^{2}$

$G$ : Mass flux, $\mathrm{kg} / \mathrm{m}^{2} \cdot \mathrm{s}$

$H$ : Heaviside function

$h$ : Heat transfer coefficient, $\mathrm{W} /\left(\mathrm{m}^{2} \cdot \mathrm{K}\right)$

$i$ : i th vector

$j: \mathrm{j}$ th vector

$k$ : Thermal conductivity, $\mathrm{W} / \mathrm{m} \cdot \mathrm{K}$

$L$ : Side length of the square loop

$m$ : Embedding dimension

$M$ : Number of neighbors in state space

$N$ : Number of data

$\mathrm{Nu}$ : Nusselt number

$Q$ : Heat input, $\mathrm{W}$

$q$ : Heat flux, $\mathrm{kW} / \mathrm{m}^{2}$

$r$ : Inner radius of the tube, $0.015 \mathrm{~m}$

$\boldsymbol{R}$ : Recurrence matrix

$R a$ : Rayleigh number

$R e$ : Reynolds number

$S$ : Skewness

$t$ : Time, $\mathrm{s}$

$T$ : Temperature, ${ }^{\circ} \mathrm{C}$

$v$ : Reconstructed vector in the $m$ dimensional space

$y$ : Time series data

\section{Greek letters}

$\alpha$ : Exponent of Rayleigh number

$\beta$ : Thermal expansion coefficient, $\mathrm{K}^{-1}$

$\varepsilon$ : Threshold distance

$\theta$ : Displacement angle, ${ }^{\circ}$

$\mu$ : Viscosity of water, $\mathrm{Pa} \cdot \mathrm{s}$

$v$ : Kinematic viscosity, $\mathrm{m}^{2} / \mathrm{s}$

$\rho$ : Density, $\mathrm{kg} / \mathrm{m}^{3}$ 
$\tau$ : Delay time

\section{Superscript and Subscript}

A, B: Thermocouple position at A and B in the loop

C, D: Thermocouple position at $\mathrm{C}$ and $\mathrm{D}$ in the loop

av: Average

down: $Q$-decrease

h: Horizontal

heat: Heating section

$i$ : i th number

$n$ : $\mathrm{n}$ th number

opt: Optimum

up: $Q$-increase

v: Vertical

w: Wall

\section{Experiments}

A schematic drawing of the rectangular natural convection loop and this photograph are shown in Figs. 1(a) and 1(b), respectively. The loop made of Pyrex glass tube of inner radius $0.015 \mathrm{~m}$ close to a square in shape, in which the length of vertical branch is $0.40 \mathrm{~m}$ and the horizontal branch is $0.41 \mathrm{~m}$. Its lower and upper branches are heated at a constant heat flux and cooled at a constant wall temperature, respectively. Both its right and left branches are thermally insulated. Moreover, the loop is mounted on a pivot at the center of the loop, which allowed rotation in the vertical plane and can be adjusted after construction. The working fluid is distilled water. Heat input $Q$ ranges from 50 to 900 $\mathrm{W}$, which corresponds to the heat flux $q$ of 1.40 to $25.1 \mathrm{~kW} / \mathrm{m}^{2}$. Here, $q$ is given by $q=Q /\left(2 \pi r L_{\text {heat }}\right)$, where, $r$ is the inner radius, and $L_{\text {heat }}$ is the length of heating section. The cooling of the upper branch was made by coaxial cylindrical water cooling jacket.

The temperatures at four positions A D in Fig. 1 were measured every 0.5 s by $\Phi 0.3 \mathrm{~mm}$ K-type thermocouples. Each temperature is described as $T_{\mathrm{A}}, T_{\mathrm{B}}, T_{\mathrm{C}}$, and $T_{\mathrm{D}}$. Temperature of Locations of $\mathrm{A}$ and $\mathrm{B}$ correspond to the mixed mean temperature for a fully developed laminar profile in the cross section of the tube (Creveling, et al., 1975; Suda, 1997). $T_{\mathrm{C}}$ and $T_{\mathrm{D}}$ correspond to a cooling water temperature of the inlet and outlet of the cooling jacket, respectively. The cooling water temperature (the wall temperature) $T_{\mathrm{w}}$ controlled from 5 to $40{ }^{\circ} \mathrm{C}$ using a water temperature controller (Carry Cool 300CN) which was manufactured by As-One Co., Ltd. $T_{\mathrm{w}}$ is equal to the temperature at the position $\mathrm{C}$. The cooling water flow rate was much enough to keep the temperature change $\Delta T_{\mathrm{CD}}\left(=T_{\mathrm{D}}-T_{\mathrm{C}}\right)$ below $1{ }^{\circ} \mathrm{C}$.

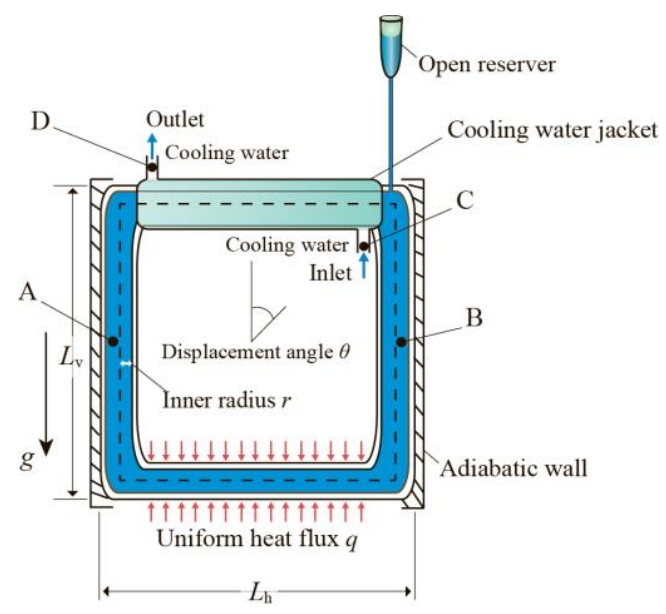

(a)

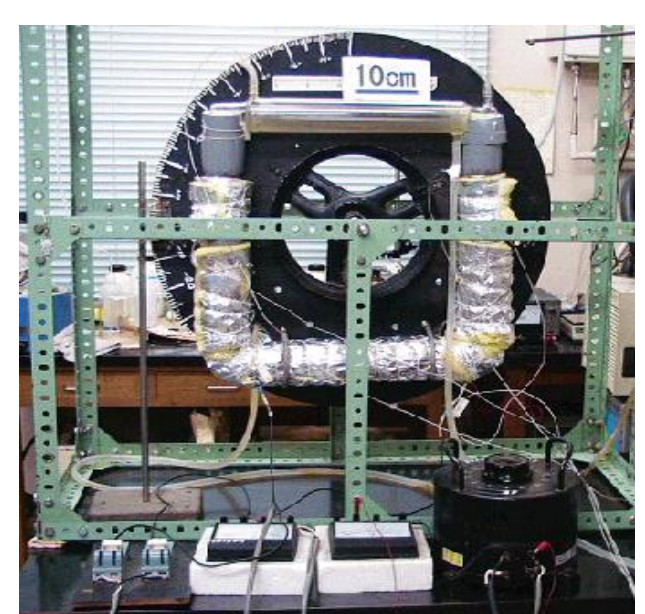

(b)

Fig. 1 Schematic drawing and a photograph of the rectangular natural convection loop. 
Thermocouple signals are stored in a data collector connected to the P.C. Heating was increased in a stepwise manner from 0 to a predetermined value. Each measurement under fixed condition takes one hundred minutes; it is sufficient time for transient effect to die out.

In the present analysis, one-dimensional time series data of the temperature difference between the positions $\mathrm{A}$ and $\mathrm{B}\left(\Delta T_{\mathrm{AB}}=T_{\mathrm{B}}-T_{\mathrm{A}}\right)$ in Fig. 1 were used.

\section{Experimental results}

\subsection{Cooling water temperature dependence of $\Delta T_{\mathrm{AB}}$ time series}

Figure 2 shows various $\Delta T_{\mathrm{AB}}$ oscillation patterns observed for several heat input $Q$ (heat flux $q$ ) and cooling water temperature $T_{\mathrm{w}}$. When the flow moves in the counter-clockwise direction, the sign of $\Delta T_{\mathrm{AB}}$ becomes positive, and vise versa. Therefore, the flow reversals can be observed through the sign changes of $\Delta T_{\mathrm{AB}}$. Figures 2 (a) (c) correspond to the case of $T_{\mathrm{w}}=5{ }^{\circ} \mathrm{C}$, (d) (f) $T_{\mathrm{w}}=25{ }^{\circ} \mathrm{C}$, and (g) (i) $T_{\mathrm{w}}=40{ }^{\circ} \mathrm{C}$. And also, Figs. 2(a), (d), (g) correspond to the case of $Q=200 \mathrm{~W}\left(q=5.51 \mathrm{~kW} / \mathrm{m}^{2}\right),(\mathrm{b}),(\mathrm{e}),(\mathrm{h})$ the case of $Q=700 \mathrm{~W}\left(19.6 \mathrm{~kW} / \mathrm{m}^{2}\right)$ and (c), (f), (i) the case of $Q=900 \mathrm{~W}(24.8$ $\mathrm{kW} / \mathrm{m}^{2}$ ). From Fig. 2, it can be seen that with increasing the $T_{\mathrm{w}}$, the flow becomes easier to be destabilized in the low $Q$ region, and the unstable flow tends to make the transition to a turbulence in the high $Q$ region. A flow state indicates a significant dependence of the cooling water temperature. We classified a flow state in the loop into four types: a laminar flow, an unstable flow, a turbulent flow and a periodical flow. In the case of $R e \leq 1200$, a flow state is the laminar flow, the case of $R e>1200$, the flow is a turbulence. A correlation between the friction factor and Reynolds number change at $R e \approx 1200$ in the rectangular loop (Suda, 1997). Our experimental results are consistent with previous studies. In the present study, when the flow reversal (the sign change of $\Delta T_{\mathrm{AB}}$ ) is observed, we defined a flow as the unstable state, regardless of the value of Reynolds number. In addition, when the periodic oscillations were observed in an autocorrelation function (i.e. power spectrum has a clear peak), the original time series was defined as the periodic oscillation (Nishikawa et al., 2008). However, as discuss in the following sections, there is a case that a different flow state occurs in the same experimental condition. Therefore, these flow states are preliminary classification.

In Figs. 2(a) (c), it was observed that a transition from a laminar to an unstable flow with increasing $Q$. Particularly, in the cases of 2(b) and 2(c), the flow reversal frequently occurs. When the flow reversal occurs, the oscillation abruptly amplifies. And also, the maximum amplitude exceeds $40{ }^{\circ} \mathrm{C}$. The flow reversal was observed in the range of $5.51 \mathrm{~kW} / \mathrm{m}^{2} \leq q \leq 24.8 \mathrm{~kW} / \mathrm{m}^{2}$. In Figs. 2(d) (f), a stable flow with a periodical change of temperature $(Q=200$ $\mathrm{W}$ and $T_{\mathrm{w}}=25^{\circ} \mathrm{C}$ ) becomes the unstable reversal flow $\left(Q=700 \mathrm{~W}\right.$ and $\left.T_{\mathrm{w}}=25^{\circ} \mathrm{C}\right)$. Then, the unstable flow transitions to the turbulence $\left(Q=900 \mathrm{~W}\right.$ and $\left.T_{\mathrm{w}}=25^{\circ} \mathrm{C}\right)$. In the case of Fig. $2(\mathrm{~g}), \Delta T_{\mathrm{AB}}$ oscillation finally reaches a stable state without the flow reversal in spite that the unstable oscillation firstly occurs. This result indicates that the flow belongs to a transitional region between the unstable flow and the turbulent flow. Figures 2(h) and 2(i) shows a developed turbulence. From these results, the unstable state of the flow is identified by observing $\Delta T_{\mathrm{AB}}$ oscillation patterns. In order to make clear the range of unstable flow state, a stability map was constructed for $T_{\mathrm{w}}$ and Reynolds number $R e$.

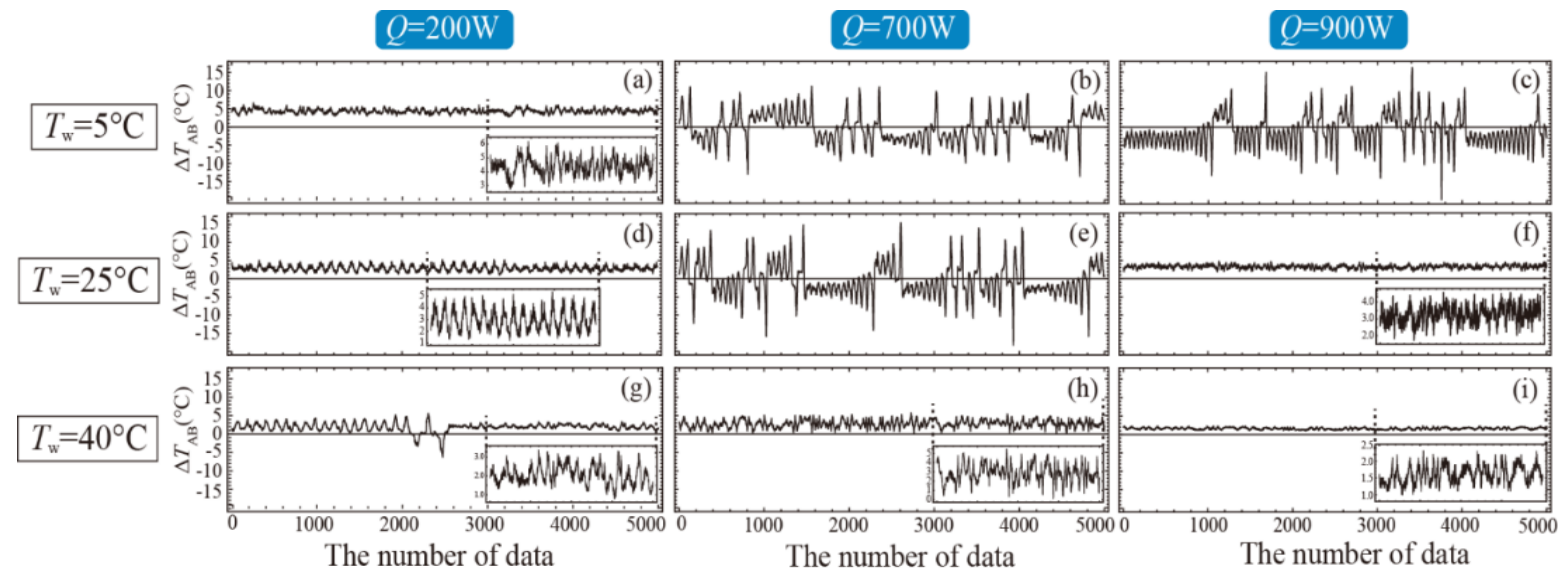

Fig. 2 Typical patterns of $\Delta T_{\mathrm{AB}}$ temperature oscillation. (a) (c), (d) (f), and (g) (i) correspond to $T_{\mathrm{w}}=5{ }^{\circ} \mathrm{C}$, $T_{\mathrm{w}}=25{ }^{\circ} \mathrm{C}$, and $T_{\mathrm{w}}=40{ }^{\circ} \mathrm{C}$, respectively. Also, (a), (d), (g) correspond to $Q=200 \mathrm{~W}\left(q=5.51 \mathrm{~kW} / \mathrm{m}^{2}\right),(\mathrm{b}),(\mathrm{e}),(\mathrm{h}) Q=700$ $\mathrm{W}\left(19.6 \mathrm{~kW} / \mathrm{m}^{2}\right)$, and (c), (f), (i) $Q=900 \mathrm{~W}\left(24.8 \mathrm{~kW} / \mathrm{m}^{2}\right)$. 


\subsection{Average characteristics of a flow state in the loop}

In this subsection, by the use of the average temperature difference $\overline{\left|\Delta T_{\mathrm{AB}}\right|}$, a stability map for the cooling water temperature $T_{\mathrm{w}}$ versus Reynolds number $R e$ is constructed. And then, a relation of Rayleigh number $R a$ against Nusselt number $N u$ was examined. Here, specific heat capacity $c$, water density $\rho$, viscosity $\mu$, thermal expansion coefficient $\beta$, thermal conductivity $k$ and heat transfer coefficient $h$ were determined by the use of the reference literature (J. P. Holman, Heat transfer, McGraw-Hill, New York, 1976, 530p). We calculated all the thermal properties as follows: firstly, the average temperature $T_{\mathrm{av}}=\left[\sum_{t=1}^{t=N}\left\{T_{\mathrm{A}}(t)+T_{\mathrm{B}}(t)\right\}\right] / 2 N$ was calculated by the temperature difference for each time series data. Secondly, the thermal properties corresponding to the average temperature is estimated by the linear or quadratic function approximation for the temperature dependence of the physical quantity.

Mass flux $G$ and $R e$ were calculated according to the following equations.

$$
\begin{aligned}
& G=\frac{Q}{\pi r^{2} c \overline{\left|\Delta T_{\mathrm{AB}}\right|}} \\
& R e=\frac{2 r G}{\mu}
\end{aligned}
$$

Figure 3 shows the flow stability map, where the filled circles correspond to the unstable flow and the filled squares to the laminar or turbulent flow, respectively. Rectangular regions enclosed by broken lines are a transitional region. Lower rectangular region of Fig. 3 represents the transition from the laminar to the unstable flow and the upper region the unstable flow to the turbulent flow. The smallest value of $R e$ is 7856 at $Q=800 \mathrm{~W}$ and $T_{\mathrm{w}}=25^{\circ} \mathrm{C}$, when the transition occurred from the unstable to the turbulent flow. In the region where both $Q$ and $T_{\mathrm{w}}$ are large, the turbulent flow is frequently observed. In the region of $R e \geq 10^{4}$, the unstable flow was not observed. In the upper transitional region, both of the unstable and the turbulent flow were observed. These cases are shown by the filled triangle in the figure. This result indicates that even when a turbulent flow is observed, a spontaneous transition to the unstable flow may occur.

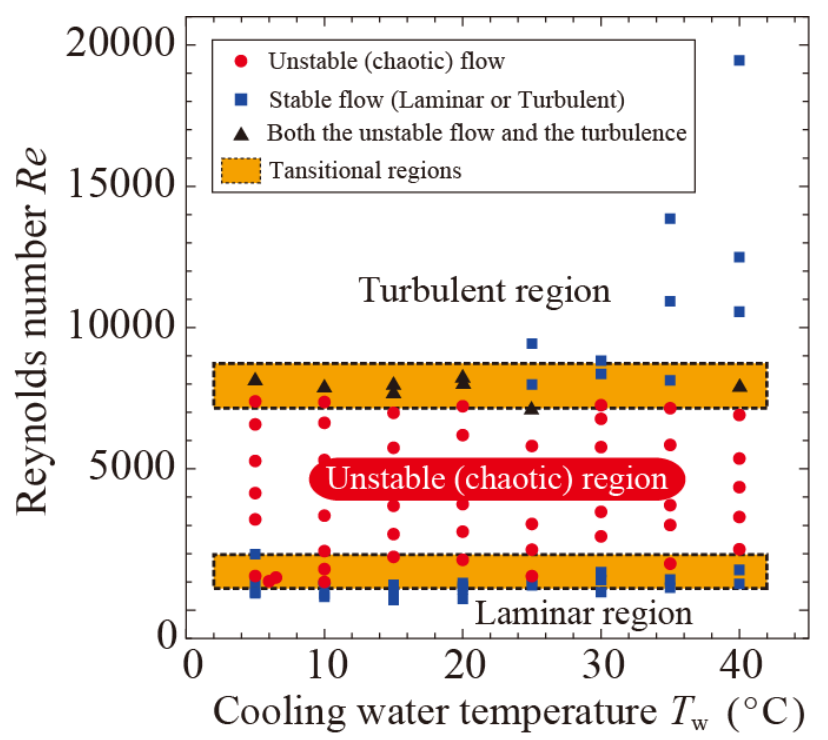

Fig. 3 Flow stability map for $R e-T_{\mathrm{w}}$ space. The filled circles and the filled squares correspond to the unstable flow and stable flows, respectively. Rectangular regions surrounded by broken lines show a transitional region. The filled triangles correspond to the case of both the unstable flow and the turbulent flow.

Next, we examined a relation between Rayleigh number and Nusselt number in order to characterize of a flow transition when increasing with heat inputs. Rayleigh number $R a$ and Nusselt number $N u$ are defined by $R a=\left\{(2 r)^{3} g \rho c \beta \Delta T_{\mathrm{AB}}\right\} / \mu k$, and $N u=(2 r h) / k$, respectively.

In Fig. 4, $N u$ are plotted against $R a$ for various heat inputs $Q$ and the cooling water temperature $T_{\mathrm{w}}$. Particularly, 
exponent $\alpha$ of $R a$ in the logarithmic plot between both non-dimensional values are estimated, and these results are shown in Table 1.

Generally, the relation between $N u$ and $R a$ is $N u \propto R a^{\alpha}$, in the soft turbulence region, $\alpha$ is $1 / 3$. When the flow is the hard turbulence, as is well known, this exponent value becomes smaller than 1/3 (Sano, et al., 1989). In the present experimental condition, there is a possibility of the transition from the soft to the hard turbulence in a region of $R a \leq 10^{7}$. Indeed, in Table 1, the exponent of $N u$ against $R a$ in the turbulent region was lower than or equal to 0.3. In Fig. 4, it was found that the range of satisfying $\alpha \leq 0.3$ becomes wider with increasing $T_{\mathrm{w}}$. This is due to the fact that a transition to a developed turbulence occur in a lower $Q$ region because the average temperature increased at high $T_{\mathrm{w}}$ conditions. The important point is that, not only the turbulent flow, but also the unstable flow is contained in the region of $\alpha<0.3$. These regions correspond to the transitional region from the unstable to the turbulent flow in Fig. 3.

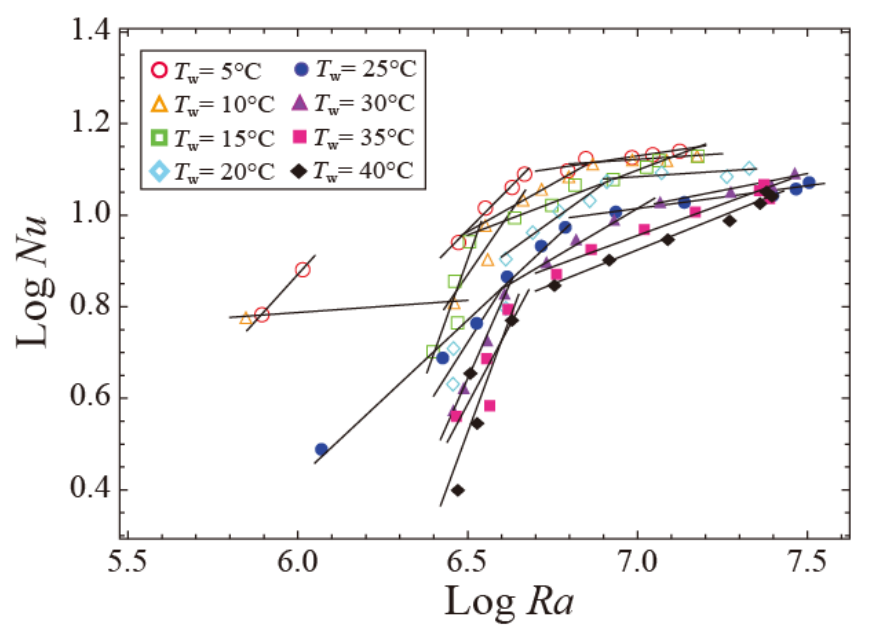

Fig. 4 Nusselt number $N u$ versus Rayleigh number $R a$. In this figure, different cases of the cooling water temperature $T_{\mathrm{w}}\left(5\right.$ to $\left.40{ }^{\circ} \mathrm{C}\right)$ are shown.

Table 1 Exponents of Rayleigh number against Nusselt number.

\begin{tabular}{|c|c|c|c|}
\hline $\begin{array}{l}\text { Cooling water } \\
\text { temperature } T_{\mathrm{w}}\left[{ }^{\circ} \mathrm{C}\right]\end{array}$ & \multicolumn{3}{|c|}{ The exponent of $R a$ number } \\
\hline 5.0 & $\begin{array}{c}0.819 \\
\left(10^{6} \leq R a \leq 10^{6.5}\right)\end{array}$ & $\begin{array}{c}0.749 \\
\left(10^{6.5} \leq R a \leq 10^{6.7}\right)\end{array}$ & $\begin{array}{c}0.111 \\
\left(10^{6.7} \leq \operatorname{Ra} \leq 10^{7.2}\right)\end{array}$ \\
\hline 10 & $\begin{array}{c}0.0533 \\
\left(10^{5.7} \leq R a \leq 10^{6.4}\right)\end{array}$ & $\begin{array}{lr}.08 & 0.41 \\
\left.R a \leq 10^{6.6}\right) & \left(10^{6.6} \leq R a\right.\end{array}$ & $\begin{array}{c}0.0496 \\
\left(10^{6.9} \leq R a \leq 10^{7.2}\right)\end{array}$ \\
\hline 15 & $\begin{array}{c}2.06 \\
\left(10^{6.4} \leq R a \leq 10^{6.6}\right)\end{array}$ & & $\begin{array}{c}0.284 \\
\left(10^{6.6} \leq R a \leq 10^{7.2}\right)\end{array}$ \\
\hline 20 & $\begin{array}{c}1.17 \\
\left(10^{6.4} \leq R a \leq 10^{6.6}\right)\end{array}$ & $\begin{array}{c}0.532 \\
\left(10^{6.6} \leq R a \leq 10^{6.9}\right)\end{array}$ & $\begin{array}{c}0.0490 \\
\left(10^{6.9} \leq R a \leq 10^{7.3}\right)\end{array}$ \\
\hline 25 & $\begin{array}{c}0.694 \\
\left(10^{6.1} \leq R a \leq 10^{6.9}\right)\end{array}$ & & $\begin{array}{c}0.0995 \\
\left(10^{6.8} \leq R a \leq 10^{7.5}\right)\end{array}$ \\
\hline 30 & $\begin{array}{c}1.67 \\
\left(10^{6.45} \leq R a \leq 10^{6.6}\right.\end{array}$ & $\begin{array}{c}0.439 \\
\left(10^{6.6} \leq R a \leq 10^{7.1}\right)\end{array}$ & $\begin{array}{c}0.146 \\
\left(10^{7.1} \leq R a \leq 10^{7.5}\right)\end{array}$ \\
\hline 35 & $\begin{array}{c}1.39 \\
\left(10^{6.45} \leq R a \leq 10^{6.6}\right)\end{array}$ & & $\begin{array}{c}0.274 \\
\left(10^{6.8} \leq R a \leq 10^{7.4}\right)\end{array}$ \\
\hline 40 & $\begin{array}{c}2.01 \\
\left(10^{6.45} \leq R a \leq 10^{6.6}\right)\end{array}$ & & $\begin{array}{c}0.300 \\
\left(10^{6.8} \leq R a \leq 10^{7.4}\right)\end{array}$ \\
\hline
\end{tabular}

From these results, following two things can be inferred: (1) although the unstable flow has a chaotic feature (e.g., an exponential extension of orbits in a phase space, and a self-similar structure of the attractor, etc.), it can be regarded as one of the turbulence state. (2) The turbulent flow in the loop has a similar dynamics of the unstable flow, i.e. a reconstructed attractor is driven by the dynamics of a few degrees of freedom (Nishikawa, et al., 2012). A temporal or spatial correlation of orbits in the phase space will collapse when the flow state becomes a more developed turbulence. This process caused by development of vortex, or development of a dynamical noise. And also, transition to developed turbulence does not occur suddenly: there is a certain width in the transitional region as already shown in Fig. 3. Consequently, an unstable chaotic feature should be detected from the turbulence data belonging to the transitional 
region. The relation of $N u$ vs. $R a$, however, was calculated using the average temperature. These values of $N u$ and $R a$ indicate only the average intensity of the convection in the loop. Therefore, in order to detect instabilities in turbulence clearly, we will evaluate the geometrical structure of a reconstructed attractor in the next section.

\section{Chaos analyses}

In this section, various chaos analyses were carried out for a scalar time series data of $\Delta T_{\mathrm{AB}}$. In the present analysis, "Chaos Analyzing System (CCI Co., Ltd.)", "Visual Recurrence Analysis" (E. Konov, 2005), and original programs written in Mathematica ver. 7.0 were used.

The following procedure was used in the present analysis. (1) An optimum delay time $\tau_{\text {opt }}$ was determined by a method of high-order autocorrelation function. (2) The attractor was reconstructed in the high-dimensional state space (Takens, 1981). (3) Recurrence plots of these attractors were constructed (Casdagli, 1997; Marwan, et al., 2002, 2007). (4) A nonlinear prediction by the method of local linear approximation was applied to the $\Delta T_{\mathrm{AB}}$ time series (Sano and Sawada, 1985; Ikeguchi and Aihara, 1995). (5) In order to evaluate the stability of the system, the recurrence plot analysis was applied to the predicted time series.

\subsection{Recurrence plot analysis}

On the basis of a scalar time series data $\Delta T_{\mathrm{AB}}, m$-dimensional state space is reconstructed using the Takens's delay method (Takens, 1981) by defining reconstructed vectors as $v(t)=(y(t), y(t+\tau), \ldots, y(t+(m-1) \tau)$, where $\tau$ is the delay time and $y(t)$ means $\Delta T_{\mathrm{AB}}(t)$. The optimum delay time $\tau_{\mathrm{opt}}$ was determined by the following process: calculating the autocorrelation function of 2 nd to 6 th order and adopting $\tau_{\mathrm{opt}}$ as the average of the first extreme values of the 4 to 6 th orders.

Recurrence plot analysis is a technique to visualize the correlation between points of distance in the reconstructed attractor (Casdagli, 1997; Marwan, et al., 2002, 2007). The main step of this method is the representation of the two-dimensional image according to the calculation of the $N \times N$ matrix,

$$
\boldsymbol{R}(i, j)=H(\varepsilon-|\boldsymbol{v}(i)-\boldsymbol{v}(j)|)
$$

where $|\cdot|$ is the Euclidean norm, and $H(\cdot)$ is the Heaviside function (i.e. $H(x)=0$ if $x<0$, and $H(x)=1$ otherwise). No. $(i$, $j)$ pixel has been represented when satisfying $\varepsilon \geq|v(i)-v(j)|$. Threshold distance $\varepsilon$ was set to 0.1 times of the maximum value of the distance between points in the reconstructed state space (Marwan, et al., 2002). The embedding dimension $m$ was set to five. Recurrence plots (RP) are an advantageous method to visualize trajectories in the state space, because typical patterns in RP structure are linked to a characteristic behavior of the system. Large scale pattern in RP can be classified in homogeneous, periodic, drift and disrupted ones (Marwan, et al., 2002, 2007). The time distance between periodic pattern corresponds to the period of orbits, and also different distance between long diagonal lines reveal quasi-periodic process. The drift is caused by the system with slowly varying parameters. This is corresponding to non-stationary systems. In this case, the short curved line may be observed, which is away from the main diagonal line $\boldsymbol{R}(i, j)=1$ in RP. And disruptions correspond to a transitional process (Marwan, et al., 2007).

Figure 5 and Fig. 6 shows a recurrence plot of typical unstable flow $\left(Q=700 \mathrm{~W}\left(q=19.6 \mathrm{~kW} / \mathrm{m}^{2}\right)\right.$ and $\left.T_{\mathrm{w}}=25^{\circ} \mathrm{C}\right)$, and a turbulence in the transitional region $\left(Q=700 \mathrm{~W}\right.$ and $\left.T_{\mathrm{w}}=40{ }^{\circ} \mathrm{C}\right)$, respectively. The enlarged view also shows in these figures. Here, $\tau_{\text {opt }}$ was 5 in the case of $Q=700 \mathrm{~W}, T_{\mathrm{w}}=25{ }^{\circ} \mathrm{C}$, and $\tau_{\text {opt }}=3$ for $Q=700 \mathrm{~W}, T_{\mathrm{w}}=40{ }^{\circ} \mathrm{C}$. Here, the optimum delay time of each data was determined by the average of the first extreme values of the 4 , 5, and 6th autocorrelation function. This value is one of a characteristic time scale of a dynamics. Generally, if a long correlation is exist in a time series, the data have a relatively large delay time. In these figures, the color of RP image is changing from yellow to dark blue color with increase in $\boldsymbol{R}(i, j)$. With increasing the distance between points in the state space, the color of RP becomes darker. In the case of $|v(i)-v(j)|$ is zero, the color is white. The maximum distance and the minimum one are different for each measurement data. Thus, the threshold $\varepsilon$ in equation (3) also different. After the distances between all vectors are calculated, they are mapped to colors of the pre-defined color map and are displayed as colored pixels in their corresponding places. Warm colors (red, yellow and orange) are associated with small distances between the vectors. Cold colors (blue, black) correspond to large distances. For example, in the case of random signals, a uniform distribution of colors over the entire image is expected. On the other hand, a deterministic 
signal will indicate a more complex or an orderly structure in the recurrence plots (E. Konov, 2005). The color gradient corresponds to the changing distance between points. Therefore, rapid changes in the rate of an expansion of the distance is expressed as a sudden change in color of RP.

Various size rectangular regions can be observed in Fig. 5. These rectangular regions are constituted by equally spaced diagonal lines, horizontal and vertical lines surrounding the diagonals. These regions will be referred as the periodic windows. In the periodic windows, diagonal lines correspond to an amplification process, horizontal and vertical lines to the flow reversals. Especially, diagonal lines reflect the deterministic dynamics. The size of the periodic window depends on the length of the diagonal lines. And also, the amplification process corresponds to the extension of the distance between the points in the state space. Therefore, as it goes to the upper right corner in the periodic windows, the color is changing darker. In Fig. 5(a), periodic windows are observed in a different observation scale. This result indicates that the reconstructed attractor of the unstable flow has characteristics of self-similarity (fractal).

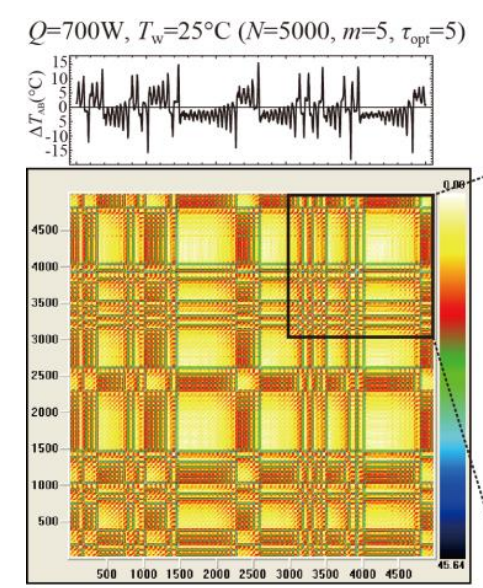

(a)

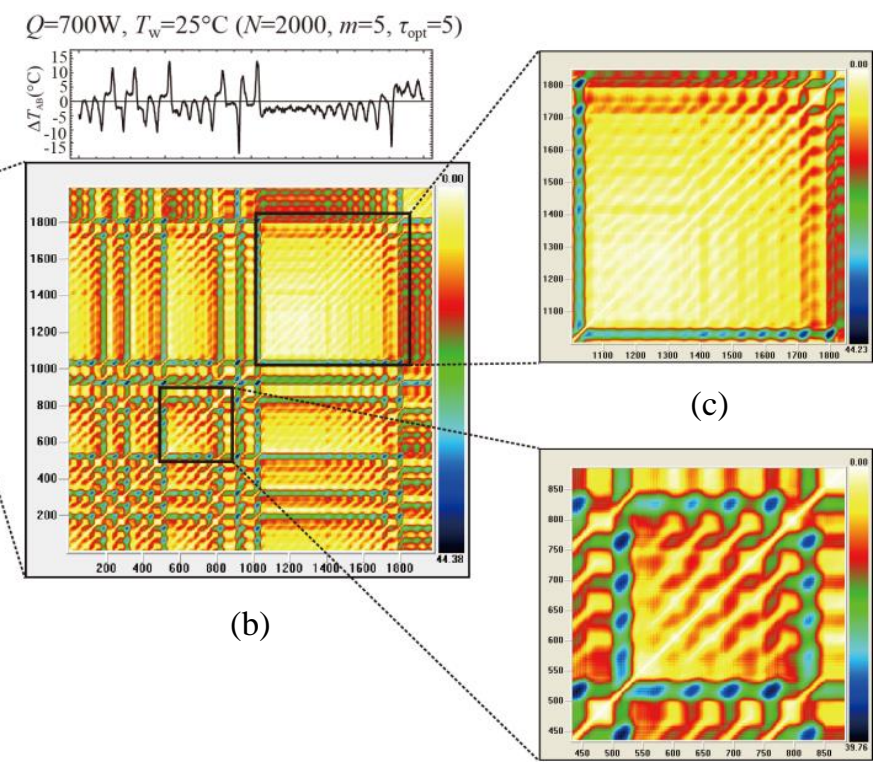

(d)

Fig. 5 An example of recurrence plot of an unstable chaotic flow. $Q=700 \mathrm{~W}\left(q=19.6 \mathrm{~kW} / \mathrm{m}^{2}\right)$ and $T_{\mathrm{w}}=25^{\circ} \mathrm{C}$.

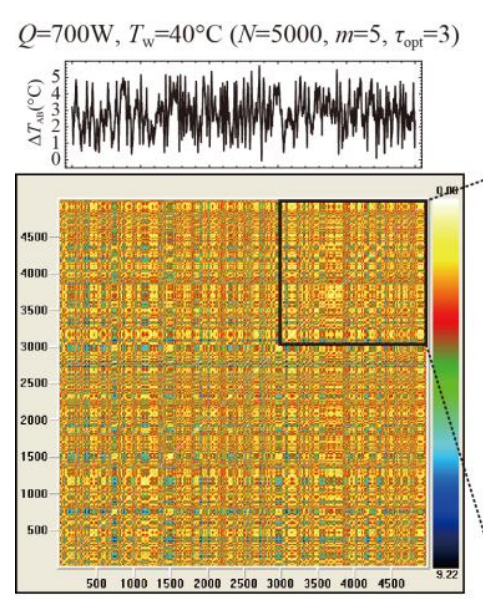

(a)

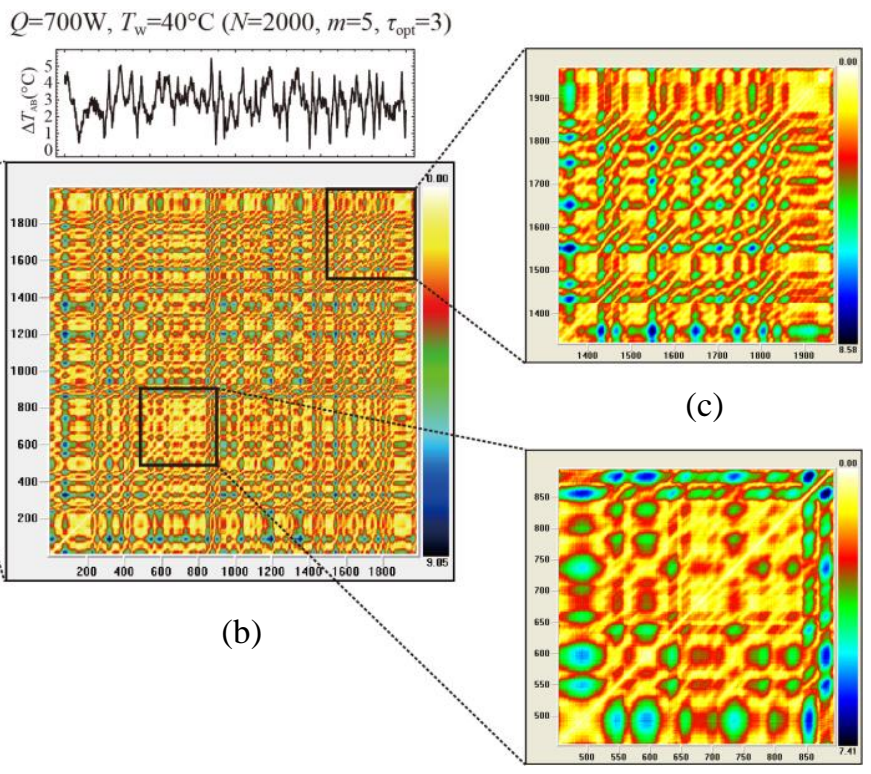

(d)

Fig. 6 An example of recurrence plot of a turbulent flow. $Q=700 \mathrm{~W}\left(q=19.6 \mathrm{~kW} / \mathrm{m}^{2}\right)$ and $T_{\mathrm{w}}=40{ }^{\circ} \mathrm{C}$. 
The periodic window is also observed in Fig. 6. In Figs. 6(c) and 6(d), it can be observed that the periodic window is composed of short lines and isolated points. If these diagonal lines occur beside single isolated points, the process could be chaotic (Marwan, et al., 2007). These recurrence plots of Fig. 6 show that there is a chaotic instability in turbulence. In other words, this fact suggests that the turbulence in the loop is very sensitive to disturbances. As the other feature, the bowed lines surrounding the main diagonal line can be seen in Fig. 6(c). These bowed line structures reflect disarray of a period structure due to a fluctuation of the temperature or the velocity in the turbulence. Hence, the bowed lines can be regarded as a kind of dislocation, which corresponds to a collapse of a periodic orbit of the attractor. If these structures are an essential feature of the turbulence, it should appear prominently in the developed turbulence.

Figure 7 shows a recurrence plot of a developed turbulent data, where Figs. 7 (a) (c) show $Q=900 \mathrm{~W}\left(24.8 \mathrm{~kW} / \mathrm{m}^{2}\right)$ and $T_{\mathrm{w}}=25^{\circ} \mathrm{C}$ and Figs. $7(\mathrm{~d}) \sim(\mathrm{f}) Q=900 \mathrm{~W}$ and $T_{\mathrm{w}}=40^{\circ} \mathrm{C}$. The enlarged view also shows in these figures. The bowed line structures can be seen clearly in the case of Figs. 7(c) and 7(f), however, the spaces of these curves are not uniform. This result is reflected that the oscillation period of the turbulence is varying. In other words, the reconstructed attractor has various recurrence orbits. The degree of development in turbulent flow can be evaluated qualitatively by the curve structures in the recurrence plot.

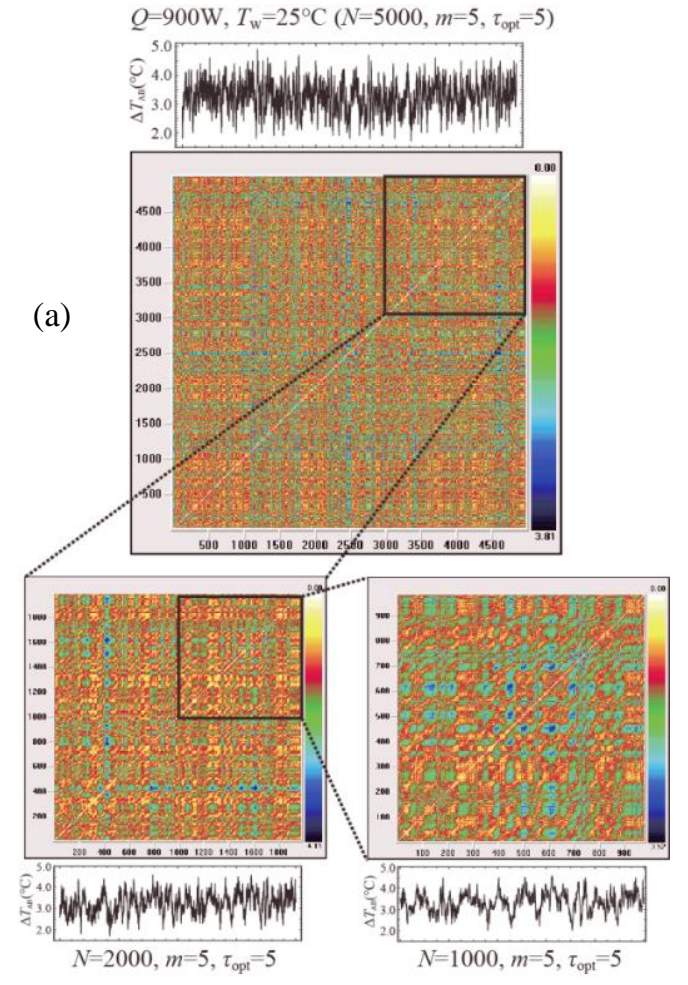

(b)

(c)

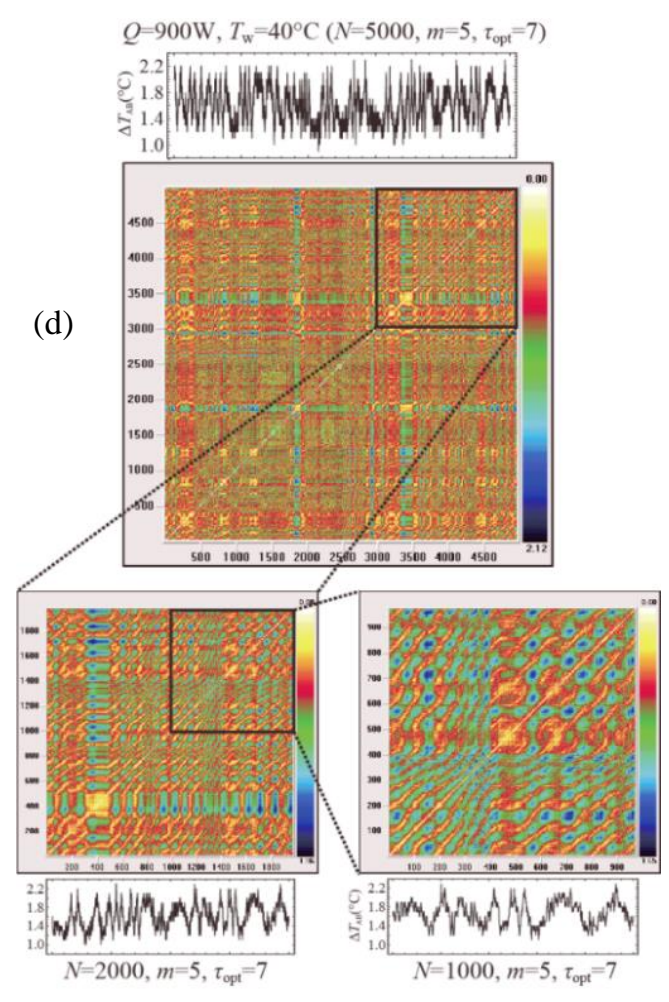

(e)

(f)

Fig. 7 Examples of recurrence plots of turbulence time series data. (a) (c): $Q=900 \mathrm{~W}$ and $T_{\mathrm{w}}=25^{\circ} \mathrm{C}$. (d) (f): $Q=900 \mathrm{~W}$ and $T_{\mathrm{w}}=40^{\circ} \mathrm{C}$.

\subsection{Evaluation of flow instability using a nonlinear prediction}

In this sub-section, turbulent data in the transitional region (in Fig. 3) were examined whether or not to destabilize using a nonlinear prediction by the method of local linear approximation (Sano and Sawada, 1985; Ikeguchi and Aihara, 1995). In this method, the prediction model is configured locally in a state space. The predicted value is calculated according to the feature of the original series. This means that the predicted time series retain the dynamics of the original data. Hence, if a sign change of the predicted time series was observed such as the unstable flow in the loop, the original data also contain the chaotic instability. In addition, we examined the dependence of the predicted time series on the number of neighbors between points in the state space. And then, the predicted time series data were evaluated by the use of recurrence plot analysis.

Examples of the predicted time series and the recurrence plot are shown in Fig. 8. These cases are $Q=700 \mathrm{~W}$, $T_{\mathrm{w}}=40^{\circ} \mathrm{C}$. We examine the dependence of the predicted time series on the number $M$ of neighbors points. Figures $8(\mathrm{a})$ 
and (b) correspond to $M=7$, and (c) and (d) to $M=50$. At the predicted time series of Figs. 8(a) and (b), a reversal oscillation can be observed. Furthermore, periodic windows are observed in the RP in Fig. 8(b). On the other hand, Figs. 8(c) and (d) show a structure similar to the RP of the original turbulent data (Fig. 7). That is, both of the periodic windows and the bowed line structure is found. These results of the prediction much depend on the value of $M$, namely, when $M$ is small, the predicted is unstable. On the contrary, with increasing $M$, the predicted time series has characteristics of turbulence.

The recurrence plot of the predicted time series data obtained from the original turbulence data has characteristics similar to those of the measured of the unstable flow. This means that even when an apparent turbulent flow is observed, a transition to the unstable flow may occur. Furthermore, it suggests that the turbulent flow in the loop can be described by chaos dynamics. Subsequently, we calculated the skewness $S$ for the predicted time series of the turbulent data in the transitional region. If the predicted time series does not have a chaotic characteristics, the oscillation is expected to be almost symmetrical with respect to the average amplitude. In this case, the $S$ will be nearly zero. On the contrary, if the predicted time series contains an abrupt change in the oscillation, as seen in Figs. 8(a) and (b), $S$ will exhibit a relatively high value.

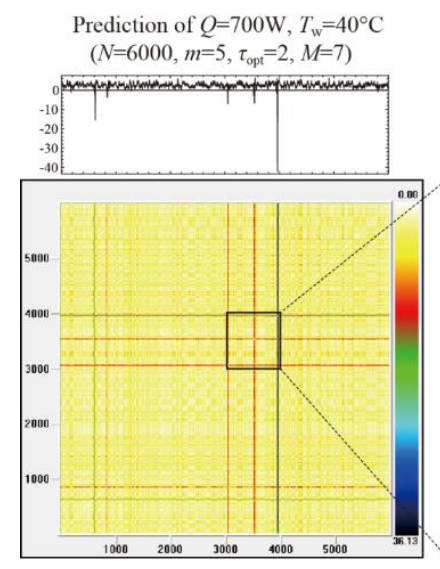

(a)

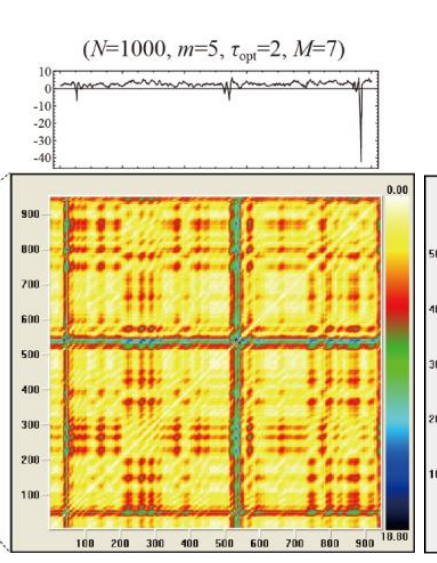

(b)

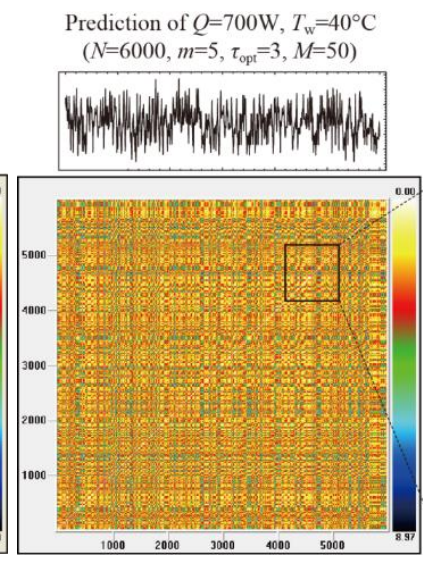

(c)

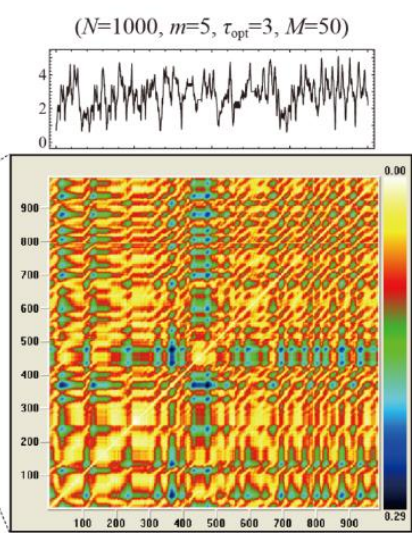

(d)

Fig. 8 Examples of the predicted time series and their recurrence plots. (a) and (b) correspond to $Q=700 \mathrm{~W}$ ( $q=19.6$ $\left.\mathrm{kW} / \mathrm{m}^{2}\right), T_{\mathrm{w}}=40^{\circ} \mathrm{C}$ and $M=7$, (c) and (d) $Q=700 \mathrm{~W}, T_{\mathrm{w}}=40^{\circ} \mathrm{C}$ and $M=50$.

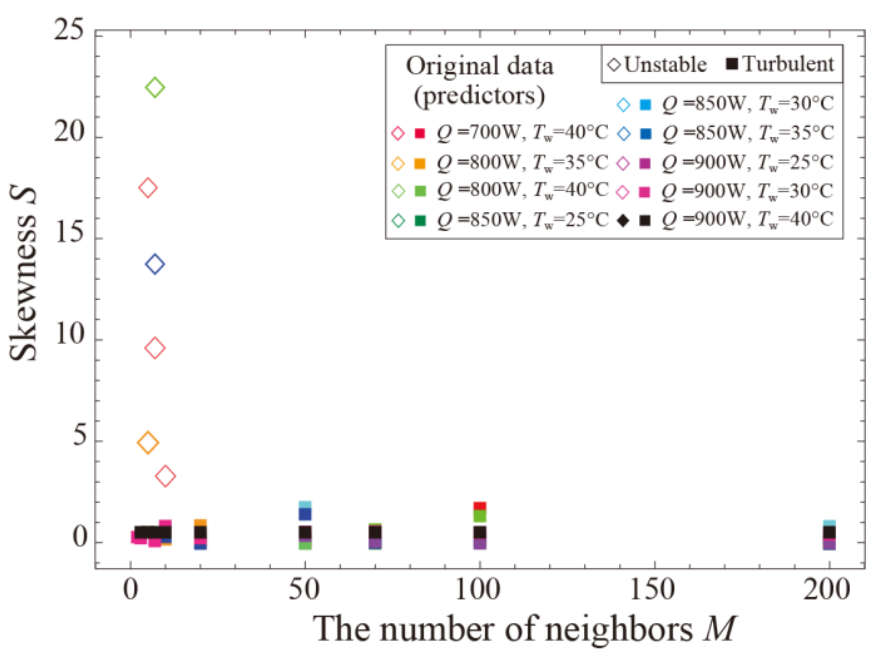

Fig. 9 Variations of skewness for predicted time series in the transitional region. Several symbols are simultaneously plotted, which correspond to the cases of varying the heat input $Q$ and the cooling water temperature $T_{\mathrm{w}}$.

In Fig. 9 shows the variations of $S$ against $M$ for the predicted time series data. Here, a predicted time series involving a sign change of the oscillation is referred to as the unstable, its represent by diamonds. And a predicted one has almost constant amplitude, which is referred to as the turbulence, it's represented by filled squares. In the case of the unstable when $M<10$, skewness is significantly greater than zero. With increasing $M$, the sign change of the 
predicted time series does not appear. Particularly, in the case of $M \geq 20$, skewness is almost zero. The results of the case of $M \leq 10$ mean that the distance between points in the reconstructed state space are significantly extend in the local scale. When $M$ is large, stretch and fold of orbits are observed about the same degree. In this case, the motion of orbits in the state space is random on average. Therefore, the instability of the system can be detected by examining the $M$ dependence of the predicted time series.

\subsection{Experimental verification of results of the chaos analysis}

From the results of the recurrence plot analysis and the nonlinear prediction, a chaotic instability has been extracted from the turbulent data in the transitional region. In order to confirm the validity of these results, we have performed another experiment with the addition of a disturbance to the loop. As the disturbance, the loop was displaced some angle. The experiment was carried out according to the following procedure. Firstly, the time series temperature data of 10000 points were measured under the condition of constant $Q$ and constant $T_{\mathrm{w}}$. Secondly, the loop was tilted for five seconds in the opposite direction to the main flow. Thirdly, the loop was returned to the horizontal position, and then, the measurement was continued. The displacement angle $\theta$ (see Fig. 1) was $5^{\circ}$, the value of which is sufficient to alter the direction of the main flow (Sata, et al., 2007).

Figure 10 shows an example of the temperature oscillation before and after adding the external disturbance under the condition of $Q=700 \mathrm{~W}$ and $T_{\mathrm{w}}=40^{\circ} \mathrm{C}$. After adding the external disturbance, the turbulent flow abruptly changed to the unstable flow. That is, the flow reversal was observed. This result indicates the turbulent flow with inherent instability, which is consistent with the results of chaos analysis. Furthermore, we confirmed that when the unstable predicted time series was obtained (this time series indicates significantly $S>0$ ), the original turbulence data has the sensitivity for the external disturbance. These results indicate that the detection of flow instability using the nonlinear prediction is effective.

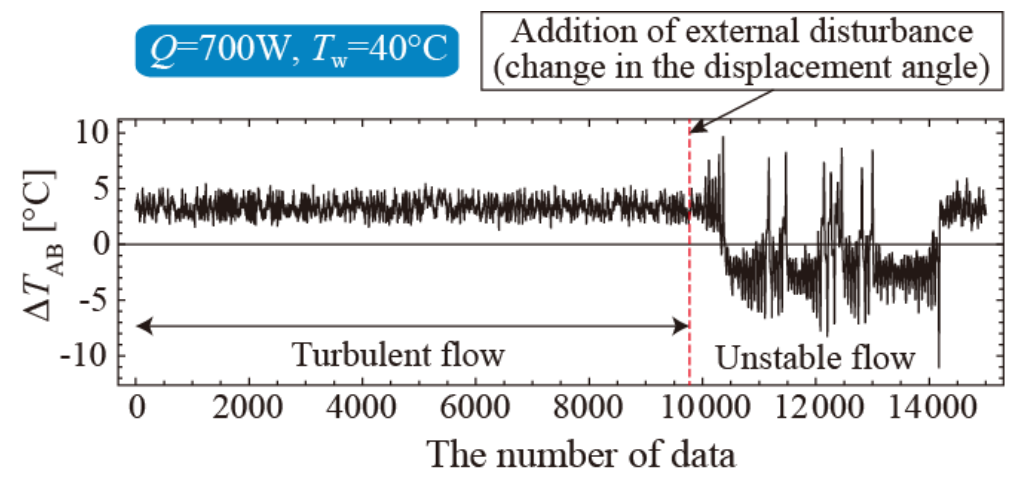

Fig. 10 An example of the experimental result with the addition of disturbance to the loop. This case is the condition of $Q=700 \mathrm{~W}\left(q=19.6 \mathrm{~kW} / \mathrm{m}^{2}\right)$ and $T_{\mathrm{w}}=40^{\circ} \mathrm{C}$.

\section{Conclusion}

In this study, the experiment for the cooling water temperature $T_{\mathrm{w}}$ dependence of the flow in the rectangular natural convection loop has been performed. Moreover, instability of the flow was evaluated using the recurrence plot analysis and the nonlinear prediction. The following results were obtained:

1) A flow state changed depending on $T_{\mathrm{w}}$. The unstable flow does not observe in the region of $R e \geq 10^{4}$. In the transitional region, however, both of the unstable and the turbulent flow are observed even when the $R e$ is the same value.

2) The recurrence plot of the turbulent flow in the transitional region shows a structure similar to the unstable chaotic flow. That is, periodic windows were observed. In addition, the bowed lines structure was also observed. This structure reflects an intensity of turbulence.

3) The unstable oscillation was predicted from the turbulent flow in the transitional region. Especially, when the number of neighbors $M$ in the state space is small, the predicted oscillation was a chaotic unstable one. When $M$ is large, it retained the characteristics of original turbulence data. 
4) We made an experiment with the addition of an external disturbance to the loop. As a result, the turbulent flow changes in the following manner: the oscillation was amplified abruptly and the flow reversal frequently occurred. This is consistent with the results of the chaos analysis.

We successfully evaluated the instability from the observed time series data. However, In order to understand the transition from the unstable to the turbulent flow, it is necessary to clarify the relationship between a spontaneous dynamical noise in the loop and the external disturbance.

\section{References}

Creveling, H. F., De Paz, J. F., Baladi, J. Y. and Schoenhals, R. J., Stability characteristics of a single-phase free convection loop, Journal of Fluid Mechanics, Vol.67, Issue 01 (1975), pp. 65-84.

Casdagli, M. C., Recurrence plots revisited, Physica D Vol.108, Issues 1-2 (1997), pp. 12-44.

Ikeguchi, T. and Aihara, K., Prediction of chaotic time series with noise. IEICE Transactions on Fundamentals of Electronics, Communications and Computer Sciences, Vol.78, No.10 (1995), pp. 1291-1298.

J. P. Holman, Heat transfer, McGraw-Hill, New York (1976), p. 530

Jiang, Y. Y. and Shoji, M., Flow stability in a natural circulation loop: influences of wall thermal conductivity, Nuclear Engineering and Design, Vol.222, Issue 1 (2003), pp.16-28.

Keller, J. B., Periodic oscillations in a model of thermal convection, Journal of Fluid Mechanics, Vol.26, Issue 03 (1966), pp.599-606.

Konov, E., VRA Software Package, Version 4.9 (2005), http://home.netcom.com/ eugenek/download.html.

Misale, M., Garibaldi, P., Passos, J. C. and de Bitencourt, G. G., Experiments in a single-phase natural circulation mini-loop, Experimental Thermal and Fluid Science Vol.31, Issue 8 (2007), pp. 1111-1120.

Misale, M., Devia, F. and Garibaldi, P., Experiments with A12O3 nanofluid in a single-phase natural circulation mini-loop: Preliminary results, Applied Thermal Engineering Vol.40 (2012), pp.64-70.

Marwan, N., Wessel, N., Meyerfeldt, U., Schirdewan, A., and Kurths, J., Recurrence-plot-based measured of complexity and their application to heart-rate-variability data, Physical Review E, 66 (2002), 026702.

Marwan, N., Romano, M. C., Thiel, M., Kurths, J., Recurrence plots for the analysis of complex systems, Physics Reports, Vol.438, Issues 5-6 (2007), pp. 237-329.

Nishikawa, H., Okino, S., and Suda, F., Chaos analysis of the stable and unstable flows in a natural convection loop: fractal dimension of time series temperature data, Journal of Advanced Science, Vol.21, No. 1\&2 (2009), pp. 20-26 (in Japanese).

Nishikawa, H., Okino, S., and Suda, F., Time Series Analysis of Flow Instability in a Natural Convection Loop, Proceedings of 9th International Conference on Flow Dynamics, Sendai (2012), pp.130-131.

Sano, M. and Sawada, Y., Measurement of Lyapunov Spectrum from a Chaotic Time Series, Physical Review Letters, 55 (1985), pp. 1082-1085.

Sano, M., Wu, X. Z. and Libchaber, A., Turbulence in helium-gas free convection, Physical Review A, Vol.40, No.11 (1989), pp.6421-6430.

Sata, Y., Wakaki, M., Okino, S., and Suda, F., Instability of the flow in a rectangular natural convection loop -effect of displacement angle and cooling water temperature on the unstable region-, Journal of Advanced Science, Vol.19, No.3\&4 (2007), pp. 29-35 (in Japanese).

Suda, F., The steady-state, transient and stability behavior of a rectangular natural convection loop -Experiments and one-dimensional model-, Experimental Heat Transfer, Fluid Mechanics and Thermodynamics (1997), pp.2191-2198.

Suda, F., Kagawa, Y., Sakabe, R. and Okino, S., Flow stability of a rectangular natural convection loop -Comparisons between water and silicone oils-, Experimental Heat Transfer, Fluid Mechanics and Thermodynamics 17 (2005), 8-b-3.

Takens, F., in Dynamical Systems and Turbulence, Warwick, 1980, edited by D. Rand and L. S. Young, Lecture Notes in Mathematics 898 (1981), pp. 366-381, Springer, Berlin.

Welander, P., On the oscillatory instability of a differentially heated fluid loop, Journal of Fluid Mechanics, Vol.29, Issue 01 (1967), pp.17-30. 